\title{
Numerical assessment of energy attraction from the sea waves by the Bristol cylinder
}

\author{
Hamid Heydari, MehranSaadatiNasab, Muhammad Shahraki*, \\ Muhammad Shojaei Pour \\ PhD student, Razi University, Kermanshah \\ Mechanic Engineering Master Student, Ferdowsi University, Mashhad \\ MSc of Chemistry, Sistan and Baluchistan University, Zahedan \\ Chemistry Engineering Master Student, Elm-o-Sanaat University
}

\begin{abstract}
Nowadays the sea waves are one of the renewable energy resources and various methods have been used for this energy resource utilization so far. In devices are set in deep waters far from the shore, the vertical parameter of wave force is the main source of energy production. Whereas in the shore nearby devices the horizontal parameter has the main role.Bristol cylinder is one of the devices which can use from both parameters effectively.In this study the Bristol cylinder operation against the regular waves is used in energy attraction.For this aim the Navier-Stokes equations have been used for the free liquid surface detection by the combining of liquid volumetric ratio.Also the virtual solvation and viscosity increasing are used for the solid object modelling.The results of the study showed that non-viscose and turbulent flow doesn't have the reasonable accuracy and the Navier-Stokes equations should be used for the modelling of Bristol cylinder, considering the viscosity and nonlinear free surface condition.
\end{abstract}

Keywords: regular waves, Bristol cylinder, Energy attractor, fluid volumetric ratio, virtual solvation domain

\section{Introduction}

Sea waves are accounted as one of the renewable energy resources nowadays and various methods have been used for the utilizing of this natural energy resource so far, as until 1980 more than thousand invention have been presented in this field.Different kind of devices for wave energy attraction work based on a buoyant or immersed object oscillation towards a constant reference.These devices efficiency could be different based on their setting depth. The Bristol cylinder is the device that can use both wave force parameters effectively, introduced for the first time by Evens (1976) in Bristol College [1]. He presented a linear theory, forecasting the devices operation and showed that the possibility of energy attraction by this device is $100 \%$ theoretically [1]. Also he revealed that for specific values of spring constant and damping coefficient of each impact wave, it is possible to remove the crossing waves and whole energy can be attracted. Recently Henkin et al (2013), upon to the potential flow theory, assessed the different parameter's effect such as elevation and the frequency of wave and also the cylinder diameter on Bristol cylinder efficiency. Davis [3] revealed empirically that linear theory losses its accuracy in computation of Bristol cylinder operation in high sharped waves. A popular method in simulation of viscosity effect consideration is the adding of a similar force term with drag force in Morison [4] relation. This method has been used by the many researchers such as Davis [3] and Barbarit et al [5]. The main problem in this method is the determination of drag coefficient. Barbarit et al showed that error in drag coefficient determination can cause the considerable uncertainty in results. Also about the Bristol cylinder, Davis [3] showed that this method couldn't present reliable results for the prediction.Due to aforementioned reasons, development of a numerical model, supposing the viscose fluid, seems necessary that assesses the real situations of Bristol cylinder operation. In this study the numerical model of Anbarsooz et al has been used for the regular nonlinear waves in real situation modelling. Also in this method the wave energy attractor operation has been simulated for different real situations.

\section{Governing equations and boundary conditions}

Schematic mechanism of wave creation by the finny wave creator has been shown in figure 1 . The solve domain, with $\mathrm{L}_{\mathrm{c}} \times \mathrm{H}_{\mathrm{c}}$ dimension, that two wave damper with $\mathrm{L} 1$ and $\mathrm{L} 2$ length have been considered. Solid object is located at $\mathrm{x}=\mathrm{Xp}$ on the left side of solve domain and creates the wave. For this aim the linear movement of finny wave creator has been simulated in solve domain. In situations that free level of fluid exists, the effect of surrounded air has been considered and the equations have been solved for the gas phase. 


\section{Governing equations on fluid current}

Governing equation on fluid current is the Navier-Stocks equation in 2 dimension, steady, compressible and Newtonian flow:

Where $\vec{V}$ is velocity vector, $\rho$ is viscosity, $\mu$ is dynamic viscosity, pis pressure, $\vec{\tau}$ stress tensor and $\overrightarrow{F_{b}}$ is the outer operated force on fluid. For the free surface of fluid detection, the fluid volumetric ratio has been used as below:

$F=\left[\begin{array}{cc}0 & \text { in the gas phase } \\ 0<,<1 & \text { in the liquid }- \text { gas interface } \\ 1 & \text { in the liquid phase }\end{array}\right.$

The effect of this quantity for each cell on density and viscosity amount is obtained based on below relations:

$\rho=F \rho_{l}+(1-F) \rho_{g}$

$\mu=F \mu_{l}+(1-F) \mu_{g}$

Where 1 and $g$ subtitles show the liquid and gas phases. In calculation steps the $\mathrm{F}$ amounts are replaced in solve domain based on below transfer equation

$$
\frac{d}{d}
$$

\section{Solid object simulation in fluid}

In this study the virtual solve domain, presented in 2005 by Sherma and Patankar [8], has been used for the finny wave maker modelling.In this method, the high viscose fluid is considered as the solid object.Pasandidehfar and Mirzaiei (2012) showed that for this aim the solid object domain viscosity should be two times greater than the fluid viscosity. In this method the solid object in solve domain is determined using the scaler quantity.

The effect of this scaler quantity entered in momentum equation via the density and viscosity changing in below equations:

For the solid object in fluid environ the governing equations of fluid solved in all calculation domain for each time step. In this step, there is no need to solve extra deferential equation for the solid object. This is an important benefit for the used method because there is no need to change the governing equations of fluid current.

In second step for the rigid movement operation on solid object, the linear and angular momentum conservation in solid object domain has been used for each time step. It means that however in first step the present velocities of solid object domain could not be calculated properly, but the mean momentum of solid 
object can be used, obtaining the mean velocity of solid object in next time step. The below integrals are used, reaching this aim.

\section{Damper and Spring Forces}

Outer operated forces on solid object movement, has been modelled, using the volumetric forces operation in momentum equation for some cells in calculation domain, located into the solid object. The outer forces in this study are the damper and spring forces, determined in each time step based on previous time step forces.

Where $\mathrm{k}_{\mathrm{sx}}$ and $\mathrm{k}_{\mathrm{sy}}$ are the spring constants, $\mathrm{c}_{\mathrm{x}}$ and $\mathrm{s}_{\mathrm{y}}$ are the damping coefficients in $\mathrm{x}$ and $\mathrm{y}$ directions respectively. The $U_{s}, V_{s}$ and $M_{s}$ are the horizontal velocity, vertical velocity and the solid object mass respectively. The $\mathrm{x}_{\mathrm{c}, \mathrm{s}}$ and $\mathrm{y}_{\mathrm{c}, \mathrm{s}}$ the location of solid object center and $\mathrm{x}_{\text {free }}$ and $\mathrm{y}_{\text {free }}$ are the spring free length in $\mathrm{x}$ and y directions respectively.

\section{Energy Attraction Efficiency}

The total operated force on cylinder contain the outer forces, spring and damper forces and the buoyancy force in addition to wave force. Upon to the Newton second law:

The energy attraction efficiency that is the ratio of average attracted power, $\bar{P}_{a b s}$, to the total average wave energy, $\bar{P}_{w}$, can be introduced by the equation 16 [9]:

\section{Initial and boundary condition}

Initial conditions of this study are the water surface without wave, constant with the velocity equals to zero. For the right, left and the under boundary of solve domain the non-slip fluid and the pressure gradient and volumetric ratio equal to zero have been considered as the boundary conditions. For the upper boundary the constant pressure equal to 1 atmosphere has been considered as the boundary condition. For the modelling of wave damping domains, the wave attraction boundary condition should be used. Based on figure 1 in this study 2 damping region have been simulated, behind the wave maker and the other is located at the end of solve domain. 


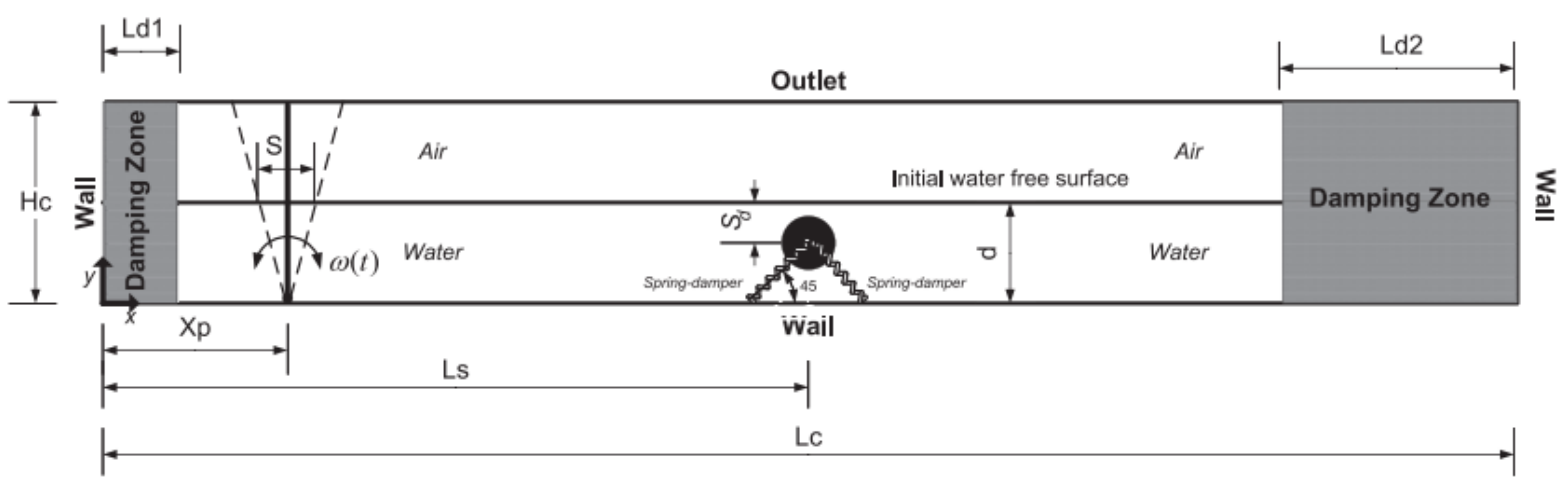

Fig1. Numerical solve domain schematic

\section{Numerical solution method}

For the governing equation discretization the Pasandidefar and Mirzaei method [7] has been utilized. This method is applicable in gridding the Euler constant for the simulation of currents with free surface and surface tension. The equations 1, 2 and 7 discretized on replaced grid. In this method the momentum equation (2) rewrite as below:

At first the intermediate velocities $\vec{V}^{*}$ are calculated based on movement, gravity and volumetric forces, using the given velocity domain of previous time step $\left(\vec{V}^{n}\right)$.

In second step, the viscosity term effect on solve domain is calculated impliedly.

Where $\overrightarrow{\tilde{V}}$ is vector of middle velocities originated from the viscosity effect. In third step, the $\overrightarrow{\widetilde{V}}$ amounts are used for pressure and velocity calculation in new time step.

Because $p^{n+1}$ and $\vec{V}^{n+1}$ are unknown, another equation is needed, for this aim the continuity equation is utilized:

Divergence of equation 16 gives the pressure Poisson's equation:

Considering that $\overrightarrow{\tilde{V}}$ has been calculated in previous step, $p^{n+1}$ is the only unknown parameter in equation that is calculated in whole solve domain. After the calculation of pressure in new time step, the velocity of new time step $\left(\vec{V}^{n+1}\right)$ is computed, using the equation 20 . 


\section{Results}

In this section the presented method capabilities, has been shown. Figure 2 shows the liquid free surface for different grids. Simulations has been applied for three 20, 40 and 80 gridding in the water depth. Considering the graph adaptions in 40 and 80 gridding, numerical solution is independent of grid and for 40 gridding has been utilized at the depth of water.

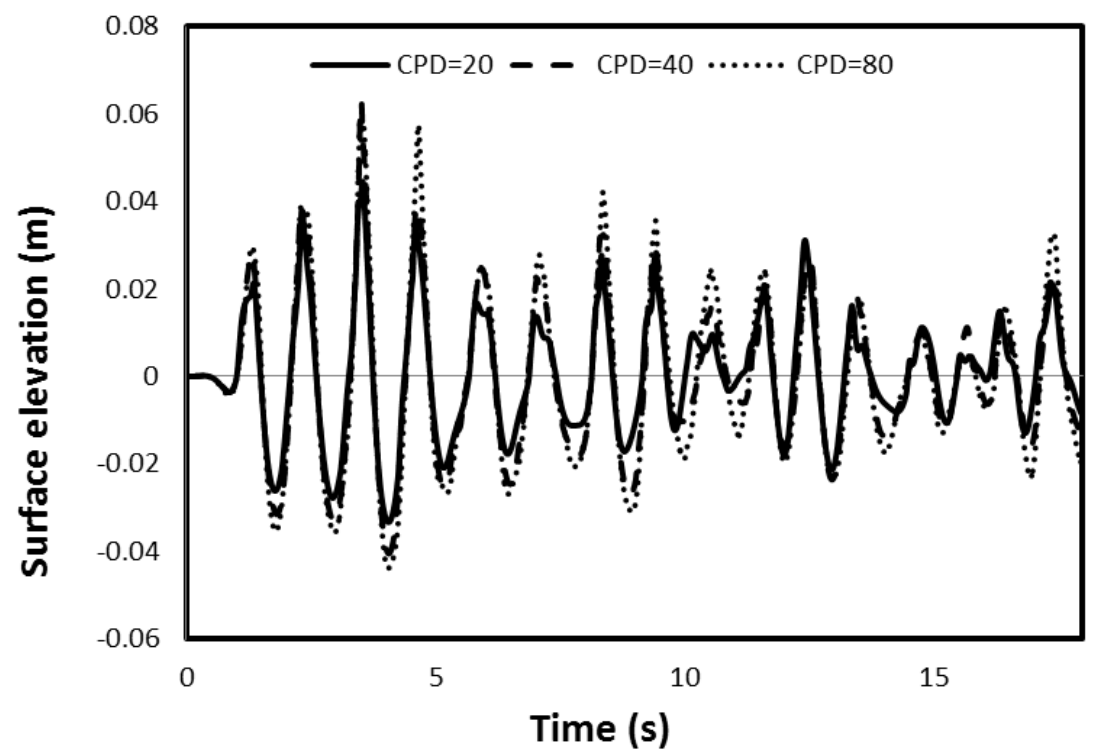

Fig2. Elevation of Wave Free level for different gridding.
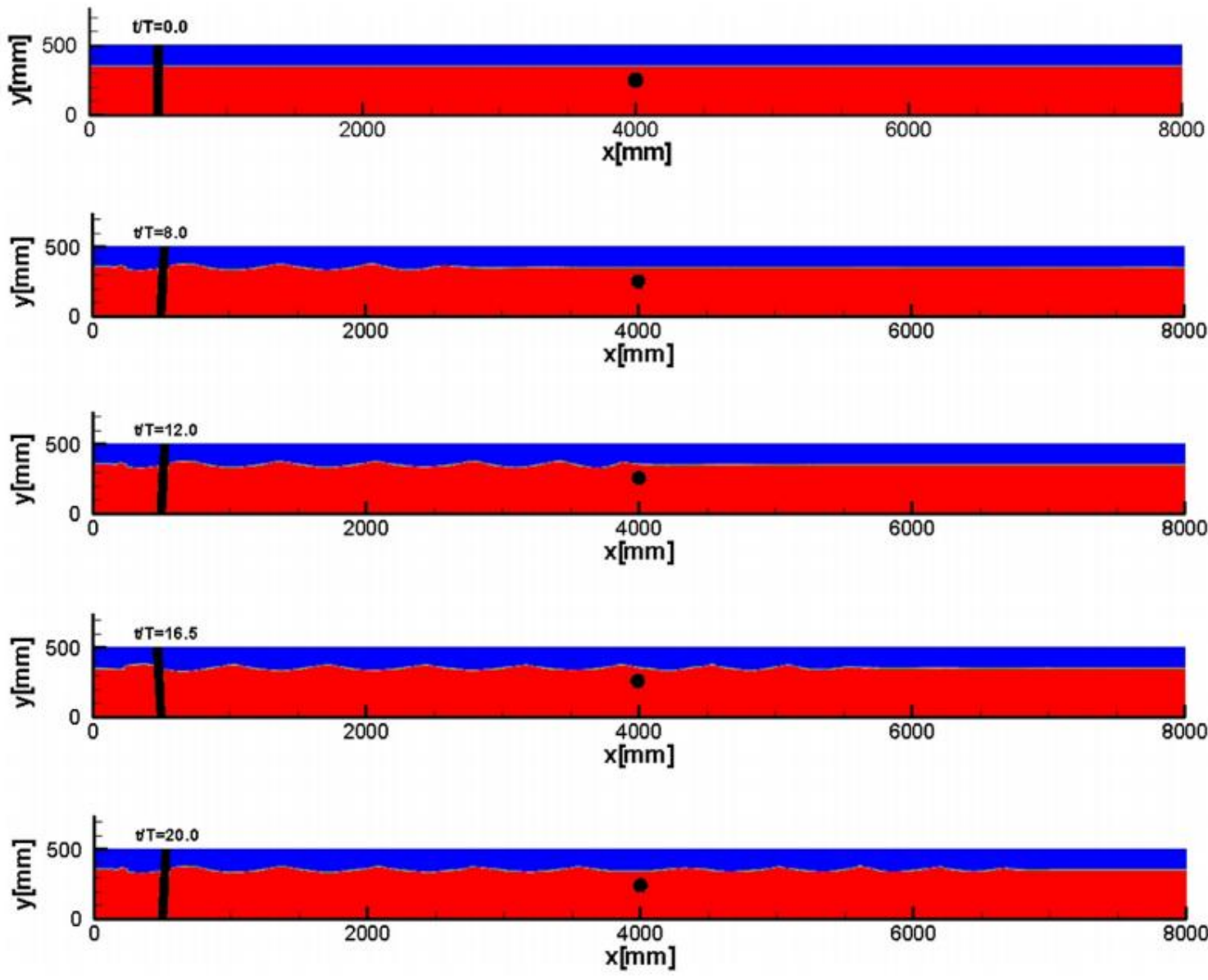

Fig3. Spatial changes of wave maker and Bristol cylinder along the time, affected by the regular waves. 
Figure 1 shows the wave maker bed towards the water free level. This bed has a finny wave maker and two damping area. Study cases are based on Davis experiments. The cylinder has been set as it has the most efficiency in 1.1 Hertz. Free water level has been shown in figure 3 along with finny wave maker movement beginning. Angular movement of wave maker has been set as the wave is made with $31 \mathrm{~mm}$ height. Upon to this movement, the attracted power by cylinder in $\mathrm{x}$ and $\mathrm{y}$ direction has been shown in figure 4 . As it can be seen the situations reach to the steady condition after the 13 periodicity. Attracted power in $y$ direction is more than $\mathrm{x}$ direction. Total power for each frequency is obtained from the sum of mean attracted power in both direction, using the equation 16. The brief properties of impacted waves on Bristol cylinder has been shown in table 1 .

Table1. Impacted wave properties on Bristol cylinder with $0.1 \mathrm{~m}$ diameter for $31 \mathrm{~mm}$ waves

\begin{tabular}{|c|c|c|c|c|c|c|c|c|}
\hline $\mathrm{KC}_{\mathrm{y}}$ & $\mathrm{KC}_{\mathrm{x}}$ & $\mathrm{P}_{\mathrm{L}}(\mathrm{W} / \mathrm{m})$ & $\mathrm{C}_{\mathrm{g}}(\mathrm{m} / \mathrm{s})$ & $\mathrm{H} / \mathrm{L}$ & $\mathrm{kR}$ & $\mathrm{kd}$ & Wave No. & Wave frequency \\
\hline $0 / 632$ & $0 / 953$ & $1 / 376$ & $1 / 376$ & $0 / 016$ & $0 / 16$ & $1 / 12$ & $3 / 19$ & $0 / 8$ \\
\hline $0 / 584$ & $0 / 729$ & $1 / 076$ & $0 / 913$ & $0 / 022$ & $0 / 221$ & $1 / 54$ & $4 / 41$ & 1 \\
\hline $0 / 516$ & $0 / 572$ & $0 / 836$ & $0 / 710$ & $0 / 030$ & $0 / 299$ & $2 / 09$ & $5 / 98$ & $1 / 2$ \\
\hline $0 / 433$ & $0 / 540$ & $0 / 678$ & $0 / 575$ & $0 / 039$ & $0 / 399$ & $2 / 79$ & $7 / 97$ & $1 / 4$ \\
\hline $0 / 345$ & $0 / 349$ & $0 / 580$ & $0 / 492$ & $0 / 051$ & $0 / 517$ & $3 / 62$ & $10 / 33$ & $1 / 6$ \\
\hline
\end{tabular}

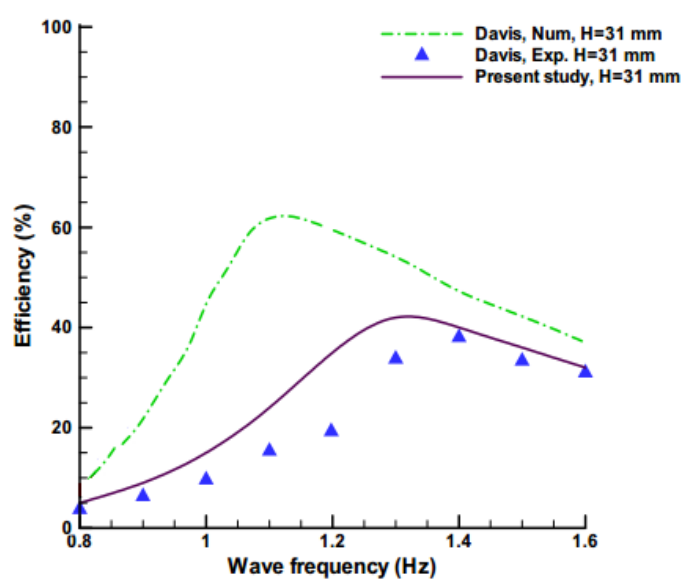

Fig5.Attracted power comparison with numerical amounts and Davis [3]

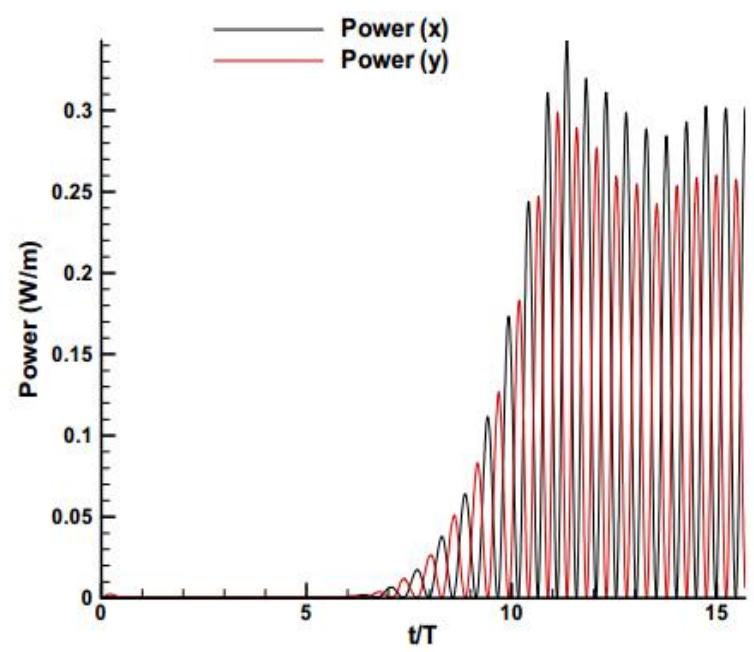

Fig4. Attracted instantaneously power by the cylinder in 2 direction $\mathrm{x}$ and $\mathrm{y}$

In figure 5 the total attracted power that has been calculated, using the numerical wave bed has been compared with numerical and empirical Davis [3] methods. Also it can be seen that however, Davis method can predict the efficiency decreasing, but there are differences between the numerical and empirical results considerably. Also Davis method couldn't predict the maximum efficiency point movementto the upper frequency. The figure 5 also shows that numerical results have the more correlation in frequency and efficiency sides. However the numerical model results has the reasonable correlation with empirical results but there is also difference between them and empirical results, that causes of spring constant and damping coefficient.

\section{References:}

[1]. Evans.D. E., A theory for wave-power absorption by oscillating bodies, Journal of Fluid Mechanic, 77 (1976) 1-25.

[2]. Heikkinen.H.,Lampinen.M.J., Boling.J., Analytical study of the interaction between waves and cylindrical wave energy converters oscillating in tow modes, Renewable Energy, 50 (2013) 150-160.

[3]. Davis.J. P., Wave energy absorption by the Bristol Cylinder-linear and non-linear effects, Proceeding of the institution of Civil Engineers, part2, 89 (1990) 317-340.

[4]. Morison.J.R.,O'Brien.M.P., Johnson.J.W., Schaaf.S.A., The force exerted by surface waves on piles, Journal of petroleum Technology, 2 (1950) 149-154.

[5]. Babarit.A.,Hals.J., Muliawan.M.J., Kurniawan.A., Moam.T., Krokstad.J., Numerical benchmarking study of a selection of wave energy converters, Renewable Energy, 41 (2012) 44-63.

[6]. Anbarsooz.M.,Passandideh-Fard.M., Moghiman.M.,Numerical simulation of a submerged cylindrical wave energy converter, Renewable Energy 64 (2014) 132-143.

[7]. Mirzaii I, Passandideh-Fard M. Modeling free surfaceflows in presence of an arbitrary moving object. Int J Multiphase Flow 39 (2012) 216-242

[8]. Sharma N, Patankar NA. A fast computation technique for the direct numerical simulation of rigid particulateflows. J Comput Phys 235 (2005) 439-457

[9]. Dean RG, Dalrymple RA. Water wave mechanics for engineers and scientists. New Jersey: Prentice Hall; 1984.

[10]. Lin, P., Liu, P.L.F., Discussion of "Vertical variation of the flow across the surf zone". Coast Eng. 50 (2004), 161-164.

[11]. Hafsia, Z., Hadj, M.B., Lamloumi, H., Maalel, K.,. Internal inlet for wave generation and absorption treatment. Coast Eng. 56 (2009), 951-959. 